\title{
Analysis of building energy upgrade technologies for implementing the dual energy efficiency and demand response scheme for non-residential buildings
}

\author{
Olga Macías ${ }^{1, *}$, Sarah Noyé ${ }^{1}$, Nagore Tellado ${ }^{1}$, Ignacio Torrens ${ }^{1}$, Pablo De Agustín ${ }^{1}$, \\ Dimosthenis Tsagkrasoulis ${ }^{2}$, Mircea Bucur $^{3}$, Jo Southernwood ${ }^{4}$ \\ ${ }^{1}$ TECNALIA, Building Technologies Division, 48160 Derio, Spain \\ ${ }^{2}$ HYPERTECH, 15232 Chalandri Athina, Greece \\ ${ }^{3}$ KIWI POWER LTD, London, United Kingdom \\ ${ }^{4}$ International Energy Research Centre, Tyndall National Institute, Cork, Ireland
}

\begin{abstract}
The continuous growth of renewable energy and the transition to a more de-centralised electricity generation adds significant complexity to balance power supply and demand in the grid. These imbalances are partially compensated by demand response programs, which represent a new business opportunity in the building sector, especially for ESCOs. Including demand response to their traditional energy efficiency-based business model adds an additional revenue stream that could potentially shorten payback periods of energy renovation projects. This paper introduces this new dual-services business model, and evaluates the potential suitability of HVAC, generation and storage technologies to ensure proposed energy efficiency and flexibility goals.
\end{abstract}

\section{Introduction}

The continuous growth of renewable energy sources (RES), characterised by their intermittent behaviour, coupled with the increasing electricity demand is causing imbalances in power grids. Moreover, recent regulations are trying to lead the existing and future building stock towards higher standards of energy efficiency, where buildings will be de-centralised sources of electricity generation. Controlling the matching between supply and demand with these new added distributed energy sources will be a challenge for the grid that could be faced by promoting flexibility on the demand-side. With this objective, demand response (DR) programs are rapidly being developed.

In this scenario, new business opportunities are arising in the building sector by means of exploiting buildings' flexibility. These opportunities could be specially interesting for Energy Services Companies (ESCOs), that could complement, with DR services, their business model focused traditionally in providing energy efficiency (EE) solutions. The viability of this new business approach is evaluated within the European Project NOVICE.

* Corresponding author: olga.macias@tecnalia.com 
Aiming to provide a tool for ensuring appropriate renovation processes, the present paper identifies the most suitable technologies for facing the two main challenges: energy efficiency and flexibility.

\section{Dual services business model}

Energy Service Companies (ESCOs) provide energy services focus on reducing energy costs of their customers by means of Energy Performance Contracting (EPC). Their traditional business model is based on implementing energy efficiency measures to generate energy savings and, consequently, cost savings. This revenue stream is used to finance investments. However, in many cases, long payback periods derived from low energy savings rates and/or high required investments limit the feasibility of these projects. By contrast, if building flexibility is included as influencing factor in selecting renovation solutions an additional revenue stream is possible thanks to DR services. This is the principle of the dual services business model developed in the NOVICE project, consisting in a new model of EPCs that combine energy efficiency and energy flexibility to maximise revenue streams. The basics of this proposed model is shown in figure 1 .

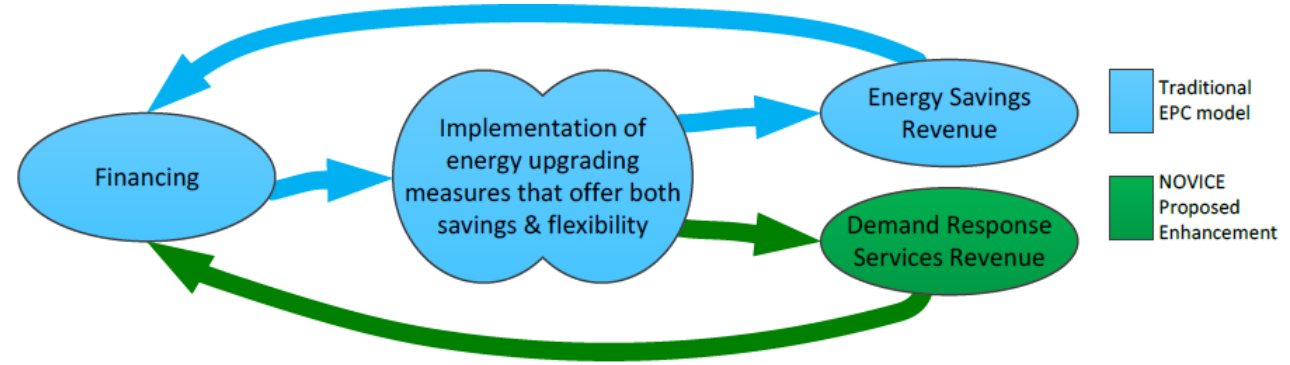

Fig. 1. Dual services business model scheme.

There are two types of DR applicable for buildings, implicit and explicit DR. Implicit DR (also called "price-based" DR) takes advantage of time-varying electricity prices, potentially reducing energy bill by shifting the energy consumption pattern to benefit from lower energy prices. Explicit DR (also called "incentive-based" DR) refers to the scheme where customers receive specific rewards to alter their consumption profile upon request triggered by high electricity demand, flexibility needs or stressed grid situations. Explicit DR is normally managed by a specific DR services provider called aggregator.

While implicit DR programs are large implemented in the global energy market, the availability of explicit DR programs depends on the DR market maturity in each country. Currently, only a few European Countries have an active and mature DR market [1]. However, the market is expected to evolve in all countries, and ESCOs now have a unique opportunity to prepare for this transformation by setting the technological foundations for the near future.

\section{Analysis of building technologies applicable for dual services}

Dual services schemes involve changes in the design of retrofitting projects. Energy solutions to be implemented must be selected considering not only EE potential, but also DR potential. This change in the renovation scope implies that ESCOs must transform their retrofitting catalogue to adapt to this new scenario. Main interventions typically implemented by ESCOs include energy efficient lighting and controls, building energy 
supply and management systems and controls [2]. Present analysis intends to identify which solutions of those typically implemented are more suitable for dual services.

Whereas, in general, lighting systems present lower potential for DR because of their high dependence with human comfort and business productivity, lighting technologies are not included in present technology review.

\subsection{HVAC technologies for dual services}

Among commercial buildings' total consumption, Heating, Ventilation and AirConditioning (HVAC) systems are the largest energy end use category [3]. This is why improvements in HVAC systems oriented both for reducing energy consumption and for increasing building flexibility have enough impact to achieve purposed goals.

From EE point of view, the main feature that has to be analysed in order to identify the most suitable solutions is the energy performance. This indicator must present high values not only for nominal conditions, but also when system operates at partial loads.

Regarding DR, suitable technologies will be those that allow the implementation of typical DR strategies in HVAC systems. At zone level main DR strategies are Global temperature adjustment and space pre-heating/cooling. At generation plant level main explicit DR strategies consist on shutting down generators for short periods of time, reducing peak loads in the grid [4]. The following table summarized dual services potential for analysed HVAC technologies.

Table 1. HVAC technologies for dual services.

\begin{tabular}{|c|c|c|c|c|}
\hline $\begin{array}{c}\text { HVAC } \\
\text { subsystem }\end{array}$ & Technology & $\begin{array}{c}\text { EE } \\
\text { potential }\end{array}$ & $\begin{array}{c}\text { DR } \\
\text { potential }\end{array}$ & $\begin{array}{c}\text { Dual services } \\
\text { potential }\end{array}$ \\
\hline \multirow{4}{*}{ Generation } & Condensing boilers & High & Low & Low \\
\cline { 2 - 5 } & $\begin{array}{c}\text { Air source / water source / } \\
\text { geothermal heat pumps }\end{array}$ & High & High & High \\
\cline { 2 - 5 } & Compression chillers & High & High & High \\
\cline { 2 - 5 } & Absorption/adsorption chillers & High & Low & Low \\
\cline { 2 - 5 } & Variable refrigerant flow systems & High & Moderate & Moderate \\
\hline \multirow{2}{*}{ Distribution } & $\begin{array}{c}\text { Variable flow pump/fans } \\
\text { Variable frequency drives }\end{array}$ & High & High & High \\
\hline \multirow{2}{*}{ Emission } & $\begin{array}{c}\text { Radiant Floor/ceiling } \\
\text { Low temperature terminal units }\end{array}$ & High & High & High \\
\cline { 2 - 5 } & High temperature terminal units & Low & High & Low \\
\hline
\end{tabular}

\subsection{On site energy generation and storage systems}

In addition to providing energy savings potential, the integration of distributed energy resources (DER) and energy storage systems increases significantly building flexibility. Therefore, this kind of technologies should also play a relevant role in dual services business model.

On one hand, electricity generation and storage systems are mature technologies in DR schemes. They enable interaction with the grid at different levels, either by direct connections to the grid, or by self-consumption allowing to reduce energy demand from the electricity grid. 
On the other hand, thermal energy storage systems allow to shift and reduce peak energy demands, enabling higher energy efficiencies for HVAC equipment that finally result on energy savings, and potential to take part in DR programs. These technologies are strongly recommended to complement HVAC systems in dual services scheme.

Table 2. Energy generation and storage technologies for dual services.

\begin{tabular}{|c|c|c|c|c|}
\hline Type & Technology & $\begin{array}{c}\text { EE } \\
\text { potential }\end{array}$ & $\begin{array}{c}\text { DR } \\
\text { potential }\end{array}$ & $\begin{array}{c}\text { Dual services } \\
\text { potential }\end{array}$ \\
\hline \multirow{2}{*}{$\begin{array}{c}\text { Electricity } \\
\text { generation }\end{array}$} & $\begin{array}{c}\text { Combined heat and power } \\
\text { Photovoltaic panels }\end{array}$ & High & High & High \\
\cline { 2 - 5 } & Fuel generator & Low & High & Moderate \\
\hline $\begin{array}{c}\text { Electricity } \\
\text { storage }\end{array}$ & $\begin{array}{c}\text { Electrochemical } \\
\text { Vanadium Redox batteries } \\
\text { Flyweels }\end{array}$ & High & High & High \\
\hline $\begin{array}{c}\text { Thermal } \\
\text { energy } \\
\text { generation } \\
\text { and storage }\end{array}$ & Solar thermal collectors & High & Low & Moderate \\
\cline { 2 - 5 } & $\begin{array}{c}\text { Water tanks } \\
\text { Borehole storage }\end{array}$ & High & High & High \\
\hline
\end{tabular}

\subsection{Building ICT technologies for dual services}

Beyond physical means to save energy and obtain flexibility, dual services require a set of ICT tools to enable automated communication and control. Because of the complicated nature of the energy market and the multiplicity of stakeholders, various existing standards can ensure reliability and security of dual services. OpenADR is designed to automate the DR related information exchange between utilities and energy management control systems [5]. The IEC 62746 standard [6] dictates the interfaces and communication protocols from smart grid to smart home/building/industrial and define some flexibility use cases. The Universal Smart Energy Framework (USEF) standard defines the role of the stakeholders of the energy market and how they can interact and benefit from it, including a flexibility value chain. It is also interesting to mention the OneM2M standard that aims at facilitating Machine-to-Machine integration, for example of IoT technologies using proprietary protocols [7].

For a building to take advantage of dual services, upgrade of some of its ICT infrastructure might be need. For implicit demand response, two key technologies are required to provide utilities and customers with real time information to drive demand modification based on electricity tariffs. First, Advanced Metering Infrastructure (AMIs), such as smart meters, are needed to enable remote and real-time meter data readings for dynamic pricing. Second, user communication and graphical interfaces are needed to inform the consumers on the varying tariffs, and possibly assist in the decision-making regarding the load control. There exist mature technologies to perform those roles. They are available in most recent buildings and are easy to install when not present.

Access to explicit DR schemes requires installation of further infrastructure to enable interaction with the aggregator and integration in the energy market. It requires higher degree of automation both in terms of communication and data transfer, and in terms of control of equipment's' load. Together with bidirectional and secure communication protocol based on the standards mentioned above, Home/Building Energy Management System (HEMS/BEMS) constitute the back bone of the implementation of flexibility and energy efficiency measures. 


\subsection{BEMS technologies}

A BEMS monitors the performance of building services such as HVAC, lighting systems, and other key energy consumers and ensures energy- and emission-efficient operations. HEMS are a simplified equivalent for homes that tend to have fewer complex systems than commercial buildings. Building Automation Systems (BAS) and Building Management Systems (BMS) are traditionally designed primarily to control system, however, newer BAS/BMS systems provide out of the box analysis tools and integration with third party (e.g. ESCO) which bring them closer to BEMS and facilitate retrofitting for dual services. In addition to process controls and energy management, BEMS enable integration of supportive sensors (occupancy, $\mathrm{CO} 2$, VOC, temperature, humidity or luminescence sensors) and actuators that assist with taking in consideration the preferences and comfort requirements from the user. They can be used to save energy (e.g. turn off systems when there is no occupants) and help to tailor DR intervention for better acceptance by the users.

They are two options to integrate BEMS with the energy market. It can be done locally through a DR edge hardware (the asset dispatch signal is transmitted locally by the DR hardware to the controlled asset) or remotely via a backend interface to the BEMS. In the second case, the DR strategies are implemented directly in the BEMS so that when a dispatch signal is received, it gets translated to a control sequence (e.g. lower asset load). The DR provider can be using off-the-shelf gateway compatible with Modbus or BACnet, the two most widely used BEMS standards to interface with DR, or customized library implementation if he is using bespoke edge equipment.

\section{Analysis result: Technology kits for dual services}

As a result from the present review, a set of different combinations of suitable technologies for dual services has been developed within NOVICE project [8]. A complete description of each combination is gathered in individual datasheets, in a way that it can be used as a guide for implementing dual services scheme. Starting from one specific HVAC system, ESCOs can identify which DR strategies are applicable, which energy generation and storage systems are suggested, and which ICT and BEMS technologies are required to maximize both EE and DR potential.

Each kit is organized as shown in figure 2.

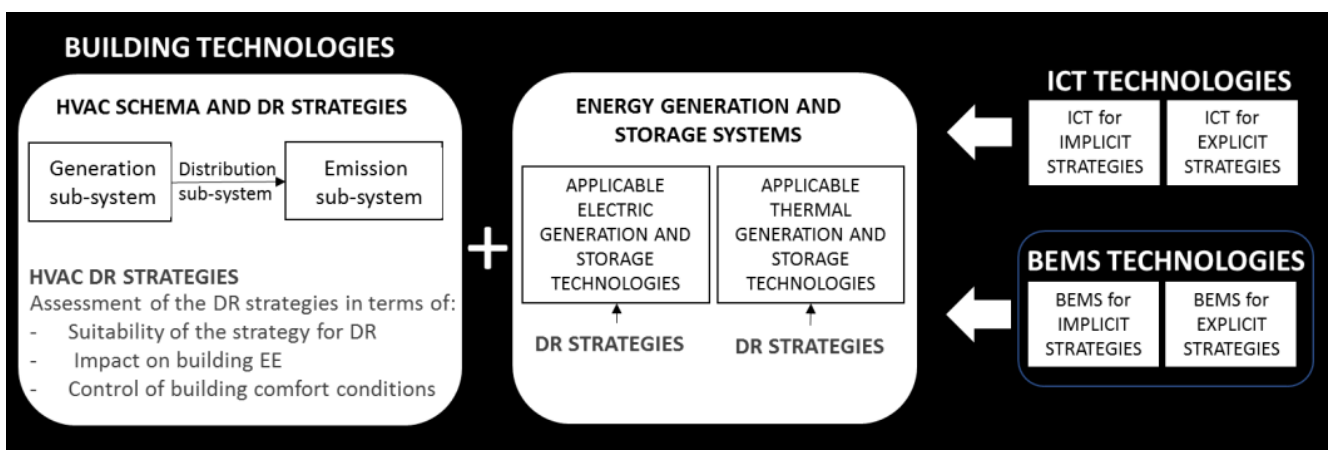

Fig. 2. Composition of technology kits developed within NOVICE project. 


\section{Conclusions}

Buildings can play a significant role in the new energy market scheme where DR programs are becoming critical solutions to face present grid challenges. In this scenario, new business opportunities are arising for ESCOs by combining traditional energy efficiency market with emerging DR market in a new business model where both EE and DR services are provided. This new business model promoted by NOVICE project enables a dual revenue stream that contributes to reduce payback periods in building renovation projects.

This approach requires the selection of appropriate building upgrade technologies to ensure improving both energy efficiency and flexibility. ESCOs must be prepared to transform their business model by including in their catalogue the suitable interventions oriented for dual services. Aiming to serve as a guide, NOVICE project provides a review of suitable technologies to be used for dual services purposes, including recommended combination of technologies to maximize profits.

The review presented in this paper concludes that HVAC systems characterized by high energy performance and using electricity as energy source are more suitable for dual services. EE and flexibility may be significantly enhanced if energy generation and storage systems are integrated. And finally, in order to supply automated communication and control for implementing both EE and DR strategies, a set of ICT tools combined with BEMS are required. Among ICT technologies, advances metering infrastructures and user communication and graphical interfaces are highlighted.

This paper is part of a project that has received funding from the European Union's Horizon 2020 research and innovation programme under grant agreement No 745594. This paper reflects only the author's views and neither the Agency nor the Commission are responsible for any use that may be made of the information contained therein.

\section{References}

1. Smart Energy Demand Coalition, Mapping Demand Response in Europe Today, SEDC (2014)

2. O. Garnier, European EPC market overview - Results of the EU-wide market survey, Deliverable 2.1, Transparence Project, UK (2013)

3. L. Pérez-Lombard, J. Ortiz, C. Pout, A review on buildings energy consumption information, Energy Build, 40 (3) 394-398, (2008)

4. D. S. Watson, S. Kiliccote, N. Motegi, M.A. Piette, Strategies for Demand Response in Commercial Buildings, Lawrence Berkeley National Laboratory, (2006)

5. OpenADR. An introduction to Automated Demand Response and the OpenADR Standard, OpenADR Alliance, (2012).

6. IEC 62762-10-1 Systems interface between customer energy management system and the power management system - Part 10-1: Open automated demand response., (2014).

7. OneM2M, OneM2M Technical specification: Functional Architecture, (2017).

8. O. Macías, S. Noye, N. Tellado, D. Tsagkrasoulis, T. Tsitsanis, M. Bucur, J. Southernwood, S. Oxisidis, Lifecycle performance of building energy upgrade technologies, Deliverable 2.1, NOVICE Project, (2018) 